\title{
Synthesis of Sultones from Chlorosulfates by a Complex Cascade Reaction Occurring under Mild Thermal Conditions
}

\author{
Lara Cala, Iván Rivilla, Fernando P. Cossío, ${ }^{*}$ Francisco J. Fañanás, and Félix Rodríguez*
}

This contribution is dedicated

\begin{abstract}
Chlorosulfate derivatives are interesting reagents that have been traditionally used to get other sulfur-containing compounds by formal nucleophilic substitution of the chlorine atom. Here, we describe a different mode of reactivity of alkyne-containing chlorosulfates to get sultones, the sulfur analogues of lactones. The complex skeletal rearrangement observed in this transformation is comparable to those intricate processes promoted or catalysed by organometallic compounds. However, the reaction here described does not require of any reagent or additive, being just a thermal process. Computational calculations support a mechanism based on a series of cascade reactions where almost every step is counterintuitive. Some of these steps include original processes related to classical reactions, thus adding complementary views to traditional organic chemistry.
\end{abstract}

The importance of sulfur-containing heterocycles is well recognised in the pharmaceutical, agrochemical, flavour and fragrance, organic materials and petrochemical industries. In this context, sultones (Figure 1), ${ }^{[1]}$ the sulfur analogues of lactones, are molecular frameworks found in many compounds with applications in areas ranging from total synthesis of natural products $^{[2]}$ to medicinal chemistry, ${ }^{[3]}$ polymers $^{[4]}$ or PETimaging. ${ }^{[5]}$ Although significant advances have been made in the synthesis of sultone derivatives, new methods to access these useful heterocycles are required.

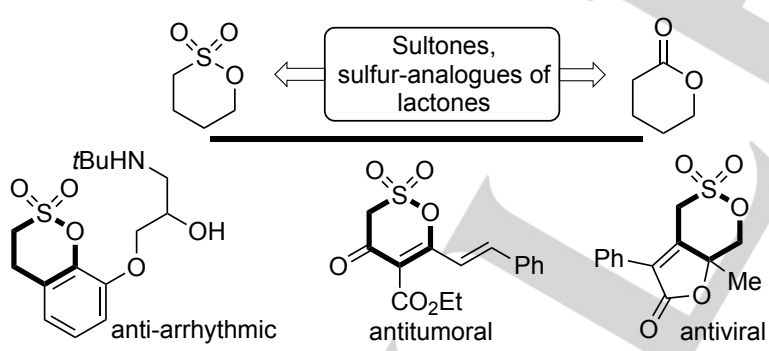

Figure 1. Sultones and lactones. Selected examples of useful sultones.

[a] Dr L. Cala, Prof. Dr F. J. Fañanás, Prof. F. Rodríguez

Instituto Universitario de Química Organometálica "Enrique Moles"

Universidad de Oviedo

Julián Clavería, 8; E-33006 Oviedo (Spain)

Fax: (+34) 985103446

E-mail: fjfv@uniovi.es; frodriguez@uniovi.es

Dr I. Rivilla, Prof. Dr F. P. Cossío

Departamento de Química Orgánica I

Universidad del País Vasco (UPV/EHU) and Donostia International

Physics Center (DIPC)

P. K. 1072; 20080 San Sebastián (Spain)

Supporting information for this article is given via a link at the end of the document.
Herein, we disclose an unusual metal-free skeletal rearrangement of simple chlorosulfate derivatives to produce sultones under mild thermal conditions by means of an intricate cascade process. It should be noted that most of the novel cascade reactions imply the use of complex organometallic reagents as promoters or catalysts. ${ }^{[6]}$ However, cascade reactions, such as the one here described, that proceed in the absence of any additional reagent or additive, are nowadays rare. Additionally, this new transformation is explained through a process that includes unusual pathways of traditional reactions, thus adding new vistas to classic processes.

In the course of our research program aimed at developing new catalytic cycloisomerization reactions of alkynol derivatives such as $1 \mathbf{a},{ }^{[7]}$ we required the synthesis of the clorosulfate derivative 2a (Scheme 1). This compound was easily accessible by treatment of alcohol 1a with sulfuryl chloride in the presence of pyridine. This high yielding reaction $(93 \%)$ could be performed at multigram scale $(4.6 \mathrm{~g})$. Moreover, chlorosulfate $2 \mathrm{a}$ was perfectly stable at room temperature and could be stored for long periods of time at $4{ }^{\circ} \mathrm{C}$ without observing decomposition. However, to our surprise, when an acetonitrile solution of 2a was heated at $80^{\circ} \mathrm{C}$ for 2 hours we observed its clean conversion into the totally unexpected sultone derivative $\mathbf{3} \mathbf{a}$, whose structure was unequivocally confirmed by $\mathrm{X}$-ray crystallographic analysis. ${ }^{[8]}$ We also verified that this new reaction could be performed on a gram scale by easily preparing 1.3 grams of sultone $3 \mathbf{a}$ in one batch without problems $(70 \%$ yield).

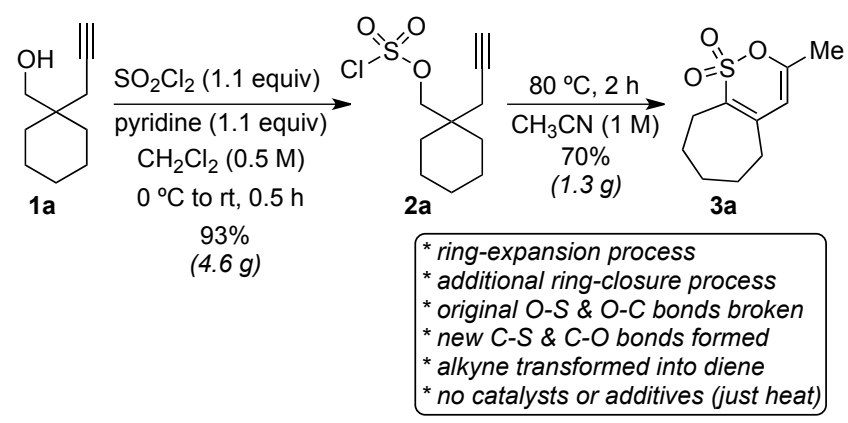

Scheme 1. Our initial experiment.

The deep skeletal rearrangement occurred in the transformation of chlorosulfate $\mathbf{2 a}$ into sultone $\mathbf{3 a}$ is worth being discussed. The original cyclohexyl moiety underwent a ring expansion process to give a seven-membered ring. An additional six-membered heterocycle was formed. Moreover, the chlorine atom was lost, the original oxygen-sulfur and carbon-oxygen bonds of $\mathbf{2 a}$ were broken and new carbon-sulfur and carbon-oxygen bonds were formed in a process where the alkyne was ultimately transformed into a conjugated diene. Although complex skeletal 
rearrangements of functionalized alkyne-containing molecules are relatively usual in the context of metal-catalysis, ${ }^{[9]}$ it should be noted that the transformation of $\mathbf{2 a}$ into sultone $\mathbf{3 a}$ occurred under mild thermal conditions without the need of any catalyst or additive. It is also important to remark that most of the known reactivity of chlorosulfate derivatives is limited to formal substitutions of the chlorine atom by appropriate nucleophiles (alcohols, amines, etc) ${ }^{[10]}$ So, in the context of chlorosulfates chemistry, the complex transformation described in this report supposes a new and unusual process. ${ }^{[11]}$

All the above-commented features of this simple and novel thermal rearrangement of chlorosulfates, along with the importance of sultones in several areas of chemistry, encouraged us to study the scope of the process. Thus, a series of chlorosulfates 2 were synthesized and then, heated at $80^{\circ} \mathrm{C}$ for 2 hours (Scheme 2). Formation of the desired bicyclic fused sultone derivatives $\mathbf{3}$ was observed in all cases. As shown, the ring expansion process comprised in this transformation allowed the synthesis of interesting sultones fused to normal-size rings (6-membered; 3b), medium-size rings (7-, and 8-membered; 3a,c,e-h) and even macrocycles (9-membered; 3d). Regarding the heterocyclic ring, only $\delta$-sultones (six-membered rings) were available, as starting materials containing longer alkyl chains linking the oxygen and the alkyne did not afford the desired sultones. However, the reaction is not limited to terminal alkynes and good results were also observed with internal ones (3e-h). commercially available chlorosulfonic acid in dry acetonitrile at $80{ }^{\circ} \mathrm{C}$ for 4 hours (Scheme 3a). At first glance, these experiments just served to identify enyne derivative $\mathbf{4 a}$ as an intermediate in the formation of sultone 3a. However, a careful analysis of the first part of this sequence (from $2 a$ to $4 a$ ) raised some intriguing mechanistic issues. Apparently, this was a simple transformation based on an alkyl shift reaction (ring expansion). However, two aspects of the reaction drew our attention. Firstly, the unusual (very mild) thermal nature of this alkyl shift, and secondly, the exclusive formation of the nonconjugated enyne derivative $4 a$ at the expenses of the alternative product $\mathbf{4} a^{\prime}$. It should be noted that alkyl shifts are usually associated to cationic processes where the initial substrate is treated with an additional reagent that promotes the formation of charged intermediates. But, in our case, formation of enyne 4a was just a thermally activated process without the participation of any external reagent.

a) Initial control experiments

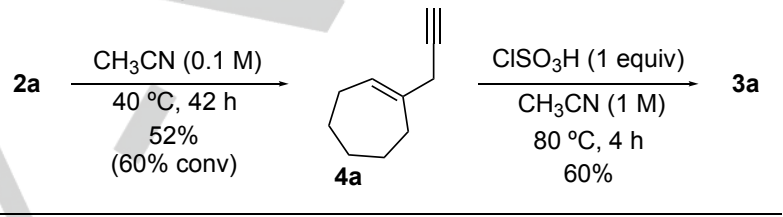

b) Alternative mechanistic proposals

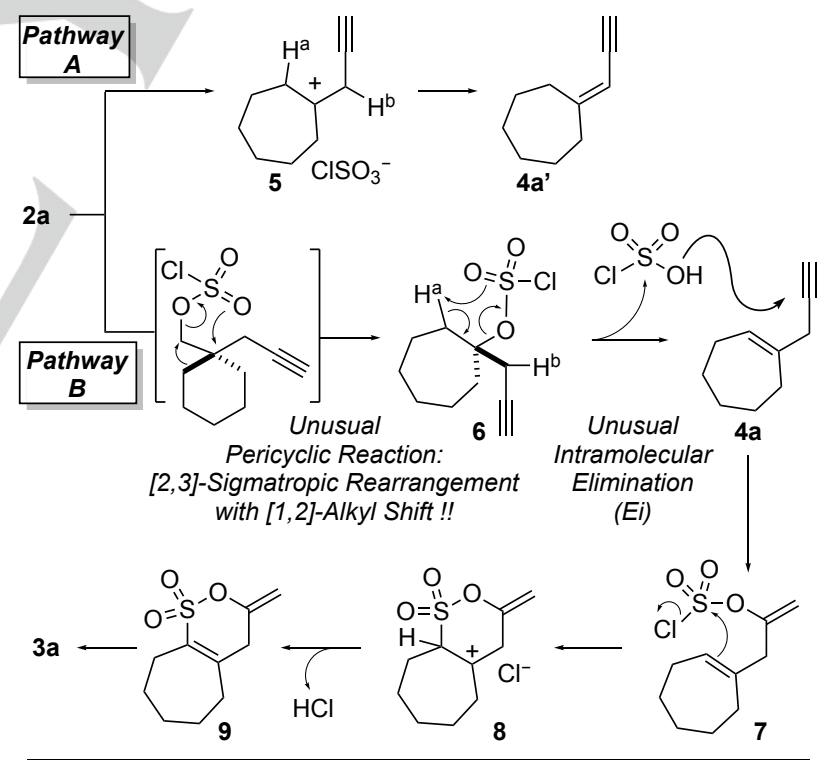

c) Additional control experiment

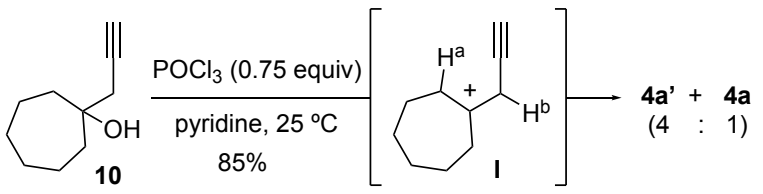

Scheme 3. Proposed mechanism for the formation of sultone derivatives 3 from chlorosulfates 2 .

Considering that the chlorosulfate group could be a good leaving group, formation of $4 a$ could be explained through a
In order to get insight into the mechanism of this reaction, we carefully examined the transformation of chlorosulfate $\mathbf{2 a}$ into sultone 3a (Scheme 3a). Thus, when a $0.1 \mathrm{M}$ solution of chlorosulfate $2 a$ in dry acetonitrile was heated at $40{ }^{\circ} \mathrm{C}$ for 42 hours $(60 \%$ conversion) we were able to isolate the enyne derivative $\mathbf{4 a}$ ( $52 \%$ yield). Interestingly, this enyne derivative was transformed into sultone $3 \mathbf{a}$ by its reaction with an equivalent of 
conventional mechanism where the elimination of the chlorosulfate group in $\mathbf{2 a}$ was favoured by the alkyl shift (ring expansion) to form the cationic intermediate 5 (Scheme 3b, pathway A). However, if this cation $\mathbf{5}$ was to be an intermediate of the process, the subsequent elimination reaction should lead to the formation of the more stable conjugated enyne derivative $4 a^{\prime}\left(H^{b}\right.$ elimination) instead of the observed non-conjugated enyne $4 a\left(H^{a}\right.$ elimination). In fact, we independently synthesized the alcohol 10 (Scheme 3c) and we observed that its treatment with the dehydrating agent $\mathrm{POCl}_{3}$ mainly led to the formation of the expected conjugated-enyne derivative $\mathbf{4 a}$ '. This reaction is supposed to proceed through a cationic intermediate I similar to 5. Considering that in our reaction from chlorosulfate derivative 2a we did not observe the formation of enyne derivative 4a', it seems unlikely that the first steps of our transformation were those shown in pathway $\mathrm{A}$ of Scheme $3 \mathrm{~b}$.

These facts encouraged us to look for alternative mechanistic proposals involving non-charged intermediates. Thus, the thermal nature of our reaction led us to consider concerted rearrangement processes (Scheme $3 b$, pathway $B$ ). Under these circumstances, we thought that starting material 2 a could evolve to form the new chlorosulfate 6 through a complex pericyclic reaction involving a [2,3]-sigmatropic rearrangement of the chlorosulfate group and a simultaneous [1,2]-alkyl shift (ring expansion). This process could be considered a single pericyclic reaction comprising two synchronic sigmatropic events. As far as we know, this is an unprecedented process in the context of "combined" pericyclic reactions. ${ }^{[12]}$ Interestingly, this reaction can also be considered an atypical formal type $I$ dyotropic rearrangement, ${ }^{[13]}$ in which one of the migrating groups (the chlorosulfate) moves through a [2,3]-shift (Scheme 4; eq. 2; $\mathrm{Z}=\mathrm{S}(\mathrm{O}) \mathrm{Cl}$ ) instead of the usual $[1,2]$-shift (Scheme 4; eq. 1).

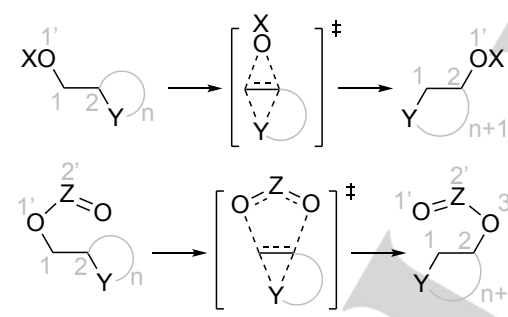

Scheme 4. Dyotropic rearrangements.

Once the new chlorosulfate 6 was formed, it could evolve to the observed non-conjugated enyne $\mathbf{4 a}$ through an intramolecular elimination $\left(E_{i}\right)$. We thought that the reaction involving the $\mathrm{H}^{a}$ hydrogen atom in 6 could be now favoured over the alternative $\mathrm{H}^{b}$-elimination. This intramolecular elimination $\left(\mathrm{E}_{\mathrm{i}}\right)$ is also remarkable. Thus, this type of reactivity is commonly found in sulfoxide, sulfone or related chemistry (i.e. Chugaev reaction) but, as far as we know, has not been reported with chlorosulfates. ${ }^{[14]}$

From intermediate $\mathbf{4 a}$, we propose that the chlorosufonic acid delivered in the previous step could add to the alkyne to form the alkenyl chlorosulfate $7 .^{[15]}$ A subsequent addition of the endocyclic alkene, through its distal carbon, to the electrophilic sulfur atom, should deliver, after elimination of chloride, the charged bicyclic intermediate 8. Conventional elimination of a proton in $\mathbf{8}$ followed by a simple isomerization of the exocyclic alkene in $\mathbf{9}$ would explain the formation of the sultone $\mathbf{3 a}$.

The sequence shown in pathway $B$ of Scheme $3 b$ included very unusual processes that seemed very attractive for computational studies. Particularly, the atypical pericyclic reactions proposed along with the addition of the chlorosulfonic acid to the alkyne and the subsequent cyclization process required some support. We performed density functional theory (DFT) calculations at the B3LYP-D3BJ(PCM=MeCN)/6-311++G(d,p) level of theory. ${ }^{[16-19]}$ Acetonitrile was used as model solvent at $80{ }^{\circ} \mathrm{C}$, thus reproducing the experimental reaction conditions. We first calculated the conventional cationic mechanism (Scheme 3b, pathway A). However, we found highly endothermic processes that required very high activation energies, non-compatible with the smooth thermal conditions under which our experiments were conducted.

a)
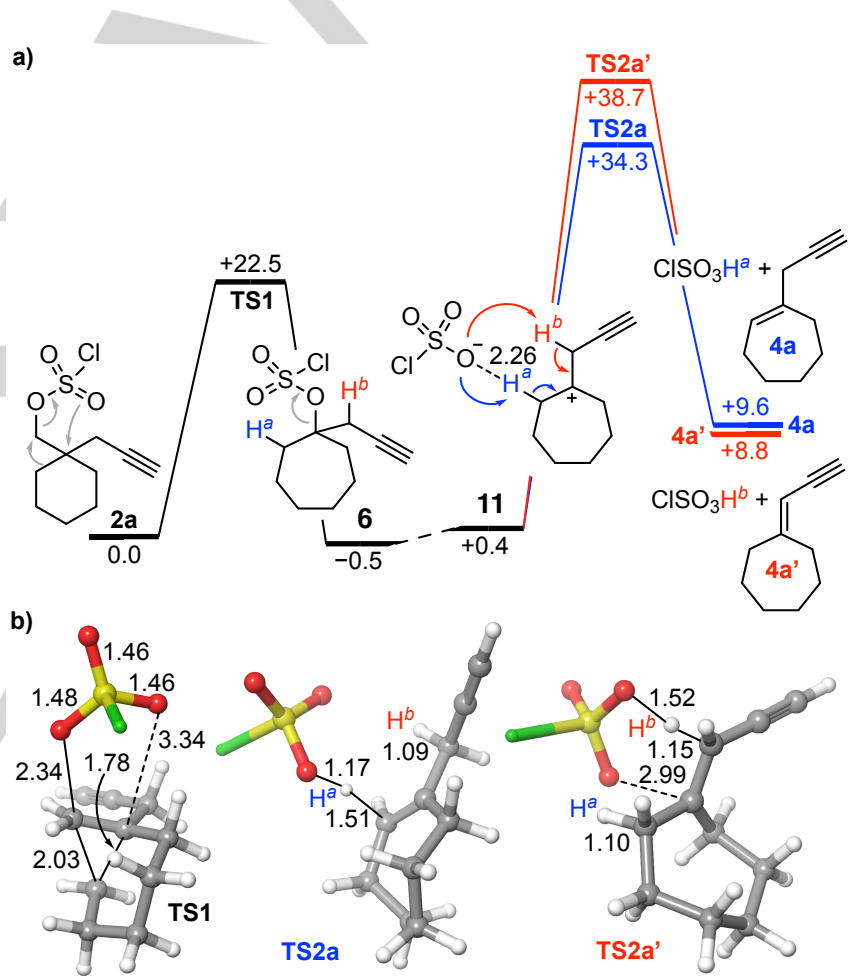

Figure 2. a) Reaction profile (B3LYP-D3BJ(PCM $=M e C N) / 6311++G(d, p)$ level of theory) associated with the transformation of reactant $2 a$ into enynes $4 a$ and 4a'. Numbers close to the stationary points correspond to the relative Gibbs energies, at $353.15 \mathrm{~K}$, in $\mathrm{kcal} / \mathrm{mol}$. b) Main geometric features of transition structures TS1, TS2a and TS2a'. Bond distances are given in $\AA$.

Instead, when we explored the reaction pathway B gathered in Scheme 3b, we located an energetically accessible transition structure TS1 (Figure 2), associated with the postulated combined ring expansion/sigmatropic shift. This saddle point carries with it an activation energy of ca. 22 $\mathrm{kcal} / \mathrm{mol}$ and is very polar, with a combined NBO charge ${ }^{[20]}$ of -0.83 a. u. for the chlorosulfonyl moiety. In addition, TS1 is very asynchronous, with a calculated synchronicity (Sy) value of only $0.68{ }^{[21]}$ This asynchronicity is due to the very different $\mathrm{C} \cdots \mathrm{O}-\mathrm{S}$ distances, which differ in ca. $1 \AA$ (Figure $2 b$ ). From this transition 
structure, the previously postulated intermediate 6 was located and conveniently characterized. Chlorosulfate 6 was calculated to be almost isoenergetic with respect to $2 \mathbf{a}$ (Figure 2a). Interestingly, the C-O bond of $\mathbf{6}$ was found to be weaker than that of $\mathbf{2 a}$. Thus, the corresponding bond distance and bond index for this bond were calculated to be $1.54 \AA$ and 0.7 in 6 , whereas the numbers for the same parameters were found to be $1.48 \AA$ and 0.8 in $\mathbf{2 a}$, respectively. As a consequence, intermediate 6 can dissociate to an almost isoenergetic (in terms of Gibbs energy at $80^{\circ} \mathrm{C}$ ) ionic pair $\mathbf{1 1}$ that showed a structure in which the anion is very close to protons $\mathrm{H}^{a}$, whereas the interaction with the two protons $\mathrm{H}^{b}$ is negligible. It seems that this close disposition of the chlorosulfate anion to $\mathrm{H}^{a}$ facilitates the subsequent abstraction of this hydrogen to give enyne derivative $4 \mathbf{a}$ through transition state TS2a. The alternative abstraction of $\mathrm{H}^{b}$ through TS2a' was found to be less favoured by $4.4 \mathrm{kcal} / \mathrm{mol}$. It is important to note that intrinsic reaction coordinate (IRC) studies on both saddle points TS2a and TS2a' led to ionic pair 11. As expected, conjugated enyne 4a' was found to be thermodynamically more stable than the nonconjugated derivative $\mathbf{4 a}$. However, the pathway leading to $\mathbf{4 a}$ ' is clearly unfavoured, in nice agreement with our experimental results.

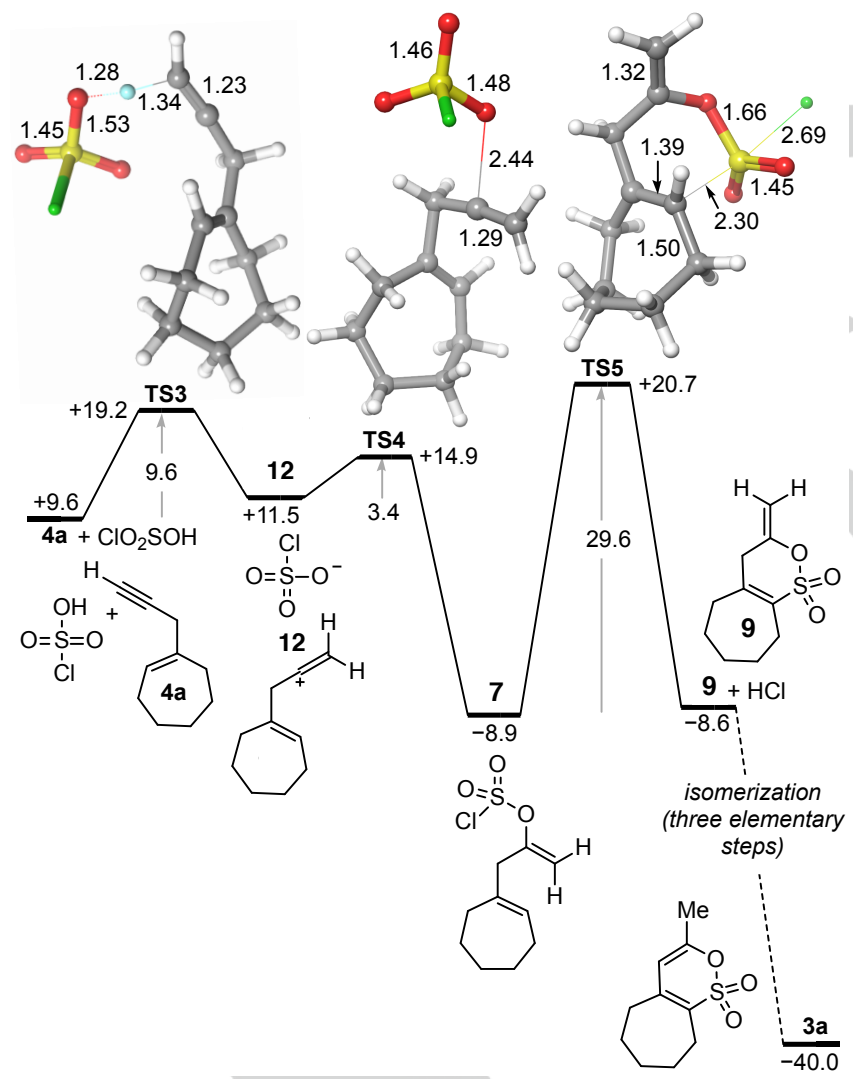

Figure 3. a) Reaction profile (B3LYP-D3BJ(PCM $=M e C N) / 6311++G(d, p)$ leve of theory) associated with the transformation of enyne $4 \mathbf{a}$ into product $3 \mathbf{a}$. Numbers close to the stationary points correspond to the relative Gibbs energies with respect to reactant 2a (See Fig. 2) at $353.15 \mathrm{~K}$, in $\mathrm{kcal} / \mathrm{mol}$. Numbers on the grey arrows are the activation free energies (at $353.15 \mathrm{~K}$, in $\mathrm{kcal} / \mathrm{mol}$ ), associated with each elementary step. The main geometric features of transition structures TS3, TS4 and TS5 are also included. Bond distances are given in $\AA$.
From enyne $\mathbf{4 a}$ and chlorosulfonic acid, the reaction path evolves towards a proton transfer on the terminal carbon atom of the alkyne moiety (Figure 3 ). This step takes place via saddle point TS3 with a quite low activation energy and gives rise to the ionic pair 12. This endergonic step collapses via transition structure TS4 to the formation of the previously proposed dienyl chlorosulfate $\mathbf{7}$, which is ca. $9 \mathrm{kcal} / \mathrm{mol}$ more stable than $\mathbf{2 a}$. From this neutral intermediate, we located and characterized saddle point TS5, which is associated with a noticeable activation energy (Figure 3 ). This cyclic transition structure exhibits a $S_{N} 2$-like geometry that involves a linear departure of the chloride anion. The IRC analysis ${ }^{[22]}$ of TS5 led to intermediate 7 , but when the loose structures generated from the forward IRC shift of TS5 were submitted to optimization, cation 8 (see Scheme $3 b$ ) collapsed with no activation barrier to bicyclic methylene $\delta$-sultone 9 . Compound $\mathbf{9}$ is almost isoenergetic with respect to chlorosulfate 7 . However, our calculations indicated that its transformation into the $\alpha, \beta$-unsaturated isomer $\mathbf{3 a}$ takes place via a downhill process involving three elementary steps associated with standard mechanisms (see the Supporting Information for additional details). Therefore, the whole transformation from $2 \mathbf{a}$ to $\mathbf{3 a}$ is strongly exergonic. This computational study supports the proposed mechanism.

In summary, an alternative mode of reactivity of chlorosulfate derivatives is reported. The process here presented escapes from the traditional reactivity of this type of compounds and supposes one of the very few recent advances in the chemistry of this functional group. More precisely, we have found that chlorosulfates derived from alkynols may be easily converted into sultone derivatives through a complex structural reorganisation that includes a ring-expansion process and the breaking and formation of several bonds. This intricate rearrangement, comparable to those processes promoted or catalysed by organometallic compounds, simply occurs by heating a solution of the starting material in acetonitrile without the need of any additional reagent or catalyst. This results in a minimal-waste, cost-efficient and operationally easy method to access $\delta$-sultones. The origins of this metal-free complex transformation have been deciphered by means of computational studies that reveal an intricate stepwise mechanism. Particularly remarkable are those steps that imply pericyclic and elimination reactions because they provide additional views into classic organic chemistry reactions.

\section{Acknowledgements}

We gratefully acknowledge financial support from Spanish Ministerio de Ciencia, Innovación y Universidades and FEDER (grants CTQ2016-76794-P, CTQ2016-80375-P and CTQ201451912-REDC, and FPU-predoctoral grant to L. C), from Consejería de Empleo, Industria y Turismo del Principado de Asturias (grant IDI/2018/000231) and from The Basque Government / Eusko Jaurlaritza (grant IT-324-07). I. R. and F. P. C. thank the SGI/IZO-SGIker UPV/EHU and DIPC for generous allocation of computational resources. 
Keywords: Density Functional Calculations • Domino Reactions - Pericyclic Reactions • Ring Expansion • Sulfur Heterocycles

[1] S. Mondal, Chem. Rev. 2012, 112, 5339-5355.

[2] Selected examples: a) P. Fischer, A. B. G. Segovia, M. Gruner, P. Metz, Angew. Chem. Int. Ed. 2005, 44, 6231-6234; Angew. Chem. 2005, 117 6387-6390; b) D. Amans, A. L. Flohic, V. Bellosta, C. Meyer, J. Cossy, Pure Appl. Chem. 2007, 79, 677-684; c) P. Fischer, M. Gruner, A Jäger, O. Kataeva, P. Metz, Chem. Eur. J. 2011, 17, 13334-13340.

[3] Selected examples: a) S. De Castro, E. Lobatón, M.-J. Pérez-Pérez, A. San Félix, A. Cordeiro, G. Andrei, R. Snoeck, E. De Clercq, J. Balzarini, M.-J. Camarasa, S. Velázquez, J. Med. Chem. 2005, 48, 1158-1168; b) S. De Castro, M. T. Peromingo, L. Naesens, G. Andrei, R. Snoeck, J. Balzarini, S. Velázquez, M.-J. Camarasa, J. Med. Chem. 2008, 51 5823-5832; c) S. De Castro, C. García-Aparicio, G. Andrei, R. Snoeck J. Balzarini, M.-J. Camarasa, S. Velázquez, J. Med. Chem. 2009, 52, 1582-1591.

[4] A. Laschewsky, Polymers 2014, 6, 1544-1601.

[5] S. Schmitt, C. Bouteiller, L. Barré, C. Perrio, Chem. Commun. 2011, 47, 11465-11467.

[6] a) L. F. Tietze, G. Brasche, K. Gericke, Domino Reactions in Organic Synthesis, Wiley-VCH, Weinheim, 2006; b) Metal Catalyzed Cascade Reactions (Ed.: T. J. J. Muller), Springer, 2006; c) D. Enders, C Grondal, M. R. M. Hüttl, Angew. Chem. Int. Ed. 2007, 46, 1570-1581; Angew. Chem. 2007, 119, 1590-1601; d) C. Grondal, M. Jeanty, D. Enders, Nat. Chem. 2010, 2, 167-178; e) Catalytic Cascade Reactions (Eds: P.-F. Xu, W. Wang), Wiley, Hoboken, 2014.

[7] For some representative works, see: a) J. Barluenga, A. Mendoza, F. Rodríguez, F. J. Fañanás, Angew. Chem. Int. Ed. 2008, 47, 7044-7047; Angew. Chem. 2008, 120, 7152-7155; b) J. Barluenga, A. Mendoza, F. Rodríguez, F. J. Fañanás, Angew. Chem. Int. Ed. 2009, 48, 1644-1647 Angew. Chem. 2009, 121, 1672-1675; c) J. Barluenga, A. Fernández, A Diéguez, F. Rodríguez, F. J. Fañanás, Chem. Eur. J. 2009, 15, 1166011667; d) F. J. Fañanás, A. Mendoza, T. Arto, B. Temelli, F. Rodríguez, Angew. Chem. Int. Ed. 2012, 51, 4930-4933; Angew. Chem. 2012, 124, 5014-5017; e) P. Pardo, A. Fernández, F. J. Fañanás, F. Rodríguez, Adv. Synth. Catal. 2012, 354, 2141-2145; f) L. Cala, A. Mendoza, F. J. Fañanás, F. Rodríguez, Chem. Commun. 2013, 49, 2715-2717; g) A. Galván, J. Calleja, F. J. Fañanás, F. Rodríguez, Angew. Chem. Int. Ed. 2013, 52, 6038-6042; Angew. Chem. 2013, 125, 6154-6158; h) J. Calleja, A. B. González-Pérez, A. R. de Lera, R. Álvarez, F. J. Fañanás, F. Rodríguez, Chem. Sci. 2014, 5, 996-1007; i) T. Arto, F. J. Fañanás, F. Rodríguez, Angew. Chem. Int. Ed. 2016, 55, 7218-7221; Angew. Chem. 2016, 128, 7334-7337.
[8] CCDC 1550282 contains supplementary crystallographic data for this paper. These data can be obtained free of charge from The Cambridge Crystallographic Data Centre via www.ccdc.cam.ac.uk/data_request/cif

[9] Paradigmatic examples of complex rearrangements of alkynecontaining molecules could be found in the context of gold-catalysis. See, for example: a) E. Jiménez-Núñez, A. M. Echavarren, Chem. Rev. 2008, 108, 3326-3350; b) A. Fürstner, Chem. Soc. Rev. 2009, 38 , 3208-3221; c) R. Dorel, A. M. Echavarren, Chem. Rev. 2015, 115, 9028-9072.

[10] See for example: a) L. S. Simpson, T. S. Widlanski, J. Am. Chem. Soc 2006, 128, 1605-1610; b) R. J. Kerns, R. J. Linhardt, Synth. Commun 1996, 26, 2671-2680.

[11] E. Buncel, Chem. Rev. 1970, 70, 323-337.

[12] T. V. Magdesieva, Russ. Chem. Rev. 2013, 82, 228-247.

[13] a) M. T. Reetz, Angew. Chem. Int. Ed. Engl. 1972, 11, 129-130; Angew. Chem. 1972, 84, 161-162; b) I. Fernández, M. F. Bickelhaupt, F. P. Cossío, Chem. Eur. J. 2012, 18, 12395-12403; c) I. Fernández, F. P. Cossío, M. A. Sierra, Chem. Rev. 2009, 109, 6687-6711.

[14] Pyrolysis of Organosulphur Compounds (Eds: S. Patai, Z. Rappoport), John Wiley \& Sons, Ltd., New York, 1993.

[15] For related additions of sulfonic acids to alkynes, see: a) A. GarciaMartinez, M. Hanack, R. H. Summerville, P. v. R. Schleyer, P. J. Stang, Angew. Chem. Int. Ed. 1970, 9, 302-303; Angew. Chem. 1970, 82, 323324; b) G. T. Crisp, A. G. Meyer, Synthesis 1994, 667-668.

[16] M. J. Frisch, et al. Gaussian 09, revision D.01; Gaussian, Inc.: Wallingford, CT, 2009 (full reference in the Supporting Information).

[17] a) A. D. Becke, J. Chem. Phys. 1993, 98, 5648-5652; b) C. Lee, W. Yang, R. G. Parr, Phys. Rev. B: Condens. Matter Mater. Phys. 1988, 37, 785-789.

[18] a) S. Grimme, J. Antony, S. Ehrlich, S. Krieg, J. Chem. Phys. 2010, 132, 154104-154104; b) S. Grimme, S. Ehrlich, L. Goerigk, J. Comput. Chem. 2011, 32, 1456-14651.

[19] a) R. Cammi, B. Mennucci, J. Tomasi, J. Phys. Chem. A. 2000, 104, 5631-5637; b) J. Tomasi, B. Mennucci, R. Cammi, Chem. Rev. 2005, 105, 2999-3094.

[20] a) J. P. Foster, F. Weinhold, J. Am. Chem. Soc. 1980, 102, 7211; b) A. E. Reed, F. Weinhold, J. Chem. Phys. 1985, 83, 1736; c) A. E. Reed, R. B. Weinstock, F. Weinhold, J. Chem. Phys. 1985, 83, 735; d) A. E. Reed, L. A. Curtiss, F. Weinhold, Chem. Rev. 1988, 88, 899.

[21] For a perfectly synchronous process, $S y=1$, see: a) P. V. R. Schleyer, J. I. Wu, F. P. Cossío, I. Fernández, Chem. Soc. Rev. 2014, 43, 49094921; b) A. Moyano, M. A. Pericas, E. Valenti, J. Org. Chem. 1989, 54, 573.

[22] a) K. Fukui, Acc. Chem. Res. 1981, 14, 363-368; b) H. P. Hratchian, H. B. Schlegel, J. Chem. Phys. 2004, 120, 9918-9924. 


\section{Entry for the Table of Contents}

\section{COMMUNICATION}

Heat it up!: Simple chlorosulfates derived from alkynols are easily transformed under mild thermal conditions into sultone derivatives through a complex cascade reaction where several bonds are broken and formed, and a ring-expansion process occurs.

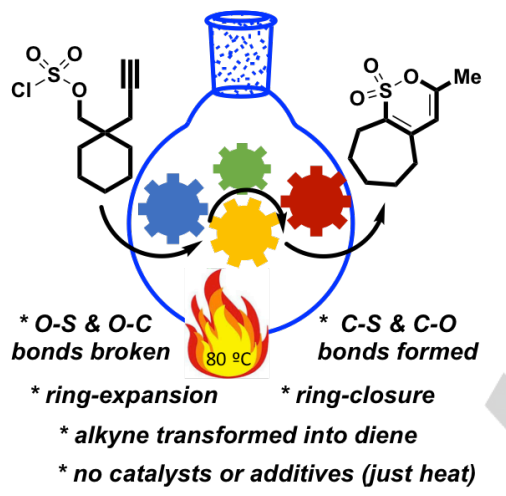

Lara Cala, Iván Rivilla, Fernando $P$. Cossio, * Francisco J. Fañanás, Félix Rodríguez*

Page No. - Page No.

Synthesis of Sultones from Chlorosulfates by a Complex Cascade Reaction Occurring under Mild Thermal Conditions 